\title{
Topical Administration Effect of Sargassum duplicatum and Garcinia mangostana Extracts Combination on Open Wound Healing Process in Diabetic Mice
}

\author{
Dwi Winarni (D), ${ }^{1}$ Fitria Nikmatul Husna, ${ }^{1}$ Muhammad Farraz Syadzha, ${ }^{1}$ \\ Raden Joko Kuncoroningrat Susilo ${ }^{D}$, ${ }^{1}$ Suhailah Hayaza, ${ }^{1}$ \\ Arif Nur Muhammad Ansori (iD, ${ }^{1,2}$ Mochammad Amin Alamsjah, ${ }^{3}$ \\ Muhamad Nur Ghoyatul Amin, ${ }^{3}$ Pugar Arga Christina Wulandari, ${ }^{4}$ Pratiwi Pudjiastuti, ${ }^{4}$ \\ and Khalijah Awang ${ }^{5}$ \\ ${ }^{1}$ Department of Biology, Faculty of Science and Technology, Universitas Airlangga, Surabaya 60115, Indonesia \\ ${ }^{2}$ Doctoral Program in Veterinary Science, Faculty of Veterinary Medicine, Universitas Airlangga, Surabaya 60115, Indonesia \\ ${ }^{3}$ Department of Marine, Faculty of Fisheries and Marine, Universitas Airlangga, Surabaya 60115, Indonesia \\ ${ }^{4}$ Department of Chemistry, Faculty of Science and Technology, Universitas Airlangga, Surabaya 60115, Indonesia \\ ${ }^{5}$ Department of Chemistry, Faculty of Science, University of Malaya, Kuala Lumpur 50603, Malaysia
}

Correspondence should be addressed to Dwi Winarni; dwi-w@fst.unair.ac.id

Received 16 June 2021; Revised 20 December 2021; Accepted 13 January 2022; Published 9 February 2022

Academic Editor: Paola Brun

Copyright (C) 2022 Dwi Winarni et al. This is an open access article distributed under the Creative Commons Attribution License, which permits unrestricted use, distribution, and reproduction in any medium, provided the original work is properly cited.

\begin{abstract}
This research aimed to determine the topical administration effect of the combination of Sargassum duplicatum and Garcinia mangostana extracts to ameliorate diabetic open wound healing. The study used 24 adult males of Mus musculus (BALB/c strain, 3-4 months, 30-40 g). They were divided into normal control groups (KN) and diabetic groups. The diabetic group was streptozotocin-induced and divided further into three treatment groups: the diabetic control group (KD), the $S$. duplicatum treatment group (PA), and the combination of $S$. duplicatum and G. mangostana treatment group (PAM). The dose of treatment was $50 \mathrm{mg} / \mathrm{kg}$ of body weight. Each group was divided into three treatment durations, which were 3 days, 7 days, and 14 days. The wound healing process was determined by wound width, the number of neutrophils, macrophages, fibroblasts, fibrocytes, and collagen density. Histological observation showed that the topical administration of combination extracts increased the reepithelialization of the wounded area, fibroblasts, fibrocytes, and collagen synthesis. The topical administration of combination extracts also decreased the number of neutrophils and macrophages. This study concluded that the topical administration of the combination of $S$. duplicatum and G. mangostana extracts improved the open wound healing process in diabetic mice.
\end{abstract}

\section{Introduction}

Diabetes mellitus (DM) is a group of metabolic disorders characterized by hyperglycemia caused by abnormalities in the process of insulin secretion, insulin performance, or both [1]. There was a prevalence of about $9.6 \%$ of the world's diabetes population in 2013, and it tends to increase until 9.9\% in 2035 [2]. Hyperglycemia is associated with increased reactive oxygen species (ROS), which triggers an increase in oxidative stress [3]. Hyperglycemia leads to the accumulation of ROS in tissues or cells with different metabolic pathways, such as activation of the polyol pathway, formation of advanced glycation end products (AGEs) and activation of its receptor, activation of isoform protein kinase C (PKC), increased activity of hexosamine pathway, and decreased antioxidant defenses. The accumulation of ROS can cause an oxidant-endogenous antioxidant imbalance which triggers the activation of sensitive intracellular signaling pathways stress, and this condition triggers cell damage and causes diabetes complications [4]. 
The wound healing process involves the following phases: the inflammatory phase (on day 3 ), the proliferation phase (on day 7), and the remodeling phase (on day 14) [5]. Various cells that play roles in wound healing include neutrophils, monocytes, macrophages, fibroblasts, B cells, T cells, mast cells, and endothelial cells [6]. Those cells are actively involved in producing and regulating various cytokines and growth factors [7]. Monocytes, which later turn into macrophages, are the primary producers of proinflammatory cytokines, such as IL- $1 \beta$ (interleukin- $1 \beta$ ), TNF$\alpha$ (tumor necrosis factor- $\alpha$ ), and IL-6 (interleukin-6). Monocytes also produce growth factors, such as VEGF (vascular endothelial growth factor), IGF-1 (insulin-like growth factor-1), and TGF- $\beta$ (transforming growth factor- $\beta$ ) in both diabetes and nondiabetes conditions, which are the essential contributors in wound healing. Neutrophils, keratinocytes, fibroblasts, mast cells, and endothelial cells are also significant producers of cytokines such as TNF- $\alpha$ and IL-10 and growth factors such as VEGF, IGF-1, and TGF- $\beta$ [6]. Diabetes leads to an impaired wound healing process, which due to the condition of hyperglycemia can increase ROS and trigger oxidative stress, thus interfering with each phase, which negatively affects the long-term quality of life, such as increased morbidity and mortality [7]. Hyperglycemia and oxidative stress change the epigenic code resulting in changes in the polarization and modulation of macrophages.

The inability to polarize macrophages is one of the main reasons the wound healing process is delayed [8]. Excessive production of proinflammatory cytokines can cause impaired angiogenic responses and microvascular complications, interfere with macrophage function and neutrophils, interfere with migration and proliferation of keratinocytes and fibroblasts, and interfere with the process of factor production growth $[9,10]$. High levels of proinflammatory cytokines in diabetes, such as IL- 6 and TNF- $\alpha$, cause disruption of the inflammatory, hyperinflammatory cascade, and insulin resistance [7]. Decreasing growth factors reduces proliferation and migration of fibrocytes and fibroblast cells. If the number of fibrocytes and fibroblasts goes down, then collagen synthesis also decreases. In addition, hyperglycemia can also inhibit the expression of endothelial nitric oxide synthase (eNOS) and increase nuclear expression factorkappa B $(\mathrm{NF}-\kappa \mathrm{B})[6]$. Decreased eNOS expression inhibits the migration of keratinocytes, thereby inhibiting the process of re-epithelialization that started at the proliferation phase. In the absence of re-epithelialization, a wound cannot be considered healed. Decreased eNOS expression also decreases VEGF so that angiogenesis is disrupted. These conditions can trigger tissue damage causing gangrene [11]. Given the lack of information about inflammatory cells in the wound healing process by topical administration, this study will only focus on the role of inflammatory cells in open wound tissue treated by a combination of natural products.

S. duplicatum, a hydrocolloid with strong absorption, absorbs a lot of rapid exudates in a gel form that creates a moist environment ideal to stimulate wound healing [10]. Alginate contains high calcium so that it can help in the process of blood clotting [11]. Moreover, alginate from S. duplicatum extracts has a polysaccharide component with a molecular weight of $3.535 \times 10^{4}$ and antioxidant activity after being assayed by the DPPH test [12]. Closing the wound using alginate can absorb excess wound exudate, maintain a humid environment physiologically, and minimize bacterial infection in the wound area [13]. G. mangostana is potentially an antioxidant that can fight free radicals played by $\alpha$-mangostin, which in the study by Wulandari et al., G. mangostana extract exhibited powerful antioxidant activity with an $\mathrm{IC}_{50}$ of 29.06 [14-17]. Therefore, this study aimed to determine the influence of a combination of S. duplicatum and G. mangostana on the wound width, the number of neutrophils, the number of macrophages, the number of fibroblasts, the number of fibrocytes, and the density of collagen in streptozotocin-induced diabetic mice.

\section{Materials and Methods}

2.1. Plant Identification. The material used in this study is the seaweed of Sargassum duplicatum and Garcinia mangostana pericarp, which were identified in the Plant Taxonomy Laboratory, Department of Biology, Universitas Airlangga, Surabaya, Indonesia.

2.2. S. duplicatum Extraction. The extraction procedures were referred to the Research and Development Center for Marine and Fisheries Product Processing and Biotechnology, Jakarta, Indonesia. Fresh S. duplicatum (50 g) was rinsed with water and added with $1.125 \mathrm{~mL}$ of $0.1 \% \mathrm{KOH}$ [18]. Then, 1\% HCL was added at a ratio of $1: 30 \mathrm{w} / \mathrm{v}$ for 1 hour and filtered. The precipitated gel was then added with $2 \% \mathrm{Na}_{2} \mathrm{CO}_{3}$ and $4 \% \mathrm{NaOCl}$. A 2-propanol solution was used to blanch the extract. After that, the extract was dried under the sun for 12 hours and then smoothened and weighed.

2.3. G. mangostana Extraction. We used the pericarp parts of the G. mangostana fruit. The pericarp was rinsed with water, blended, and macerated with ethanol. Then, the solvent was evaporated with a rotary vacuum evaporator at $50^{\circ} \mathrm{C}$. The crude extract was collected, lyophilized, and weighed [19].

2.4. Diabetic Mice Induction. This study used healthy adult male mice (Mus musculus), strain BALB/c, aged 3-4 months, with a body weight ranging from $30-40 \mathrm{~g}$. Mice were obtained from the Faculty of Pharmacy, Universitas Airlangga, Surabaya, Indonesia, and were acclimatized for two weeks in the Animal Laboratory, Faculty of Science and Technology, Universitas Airlangga, Surabaya, Indonesia. All animal procedures were carried out in compliance with the protocol approved by the Animal Care and Use Committee, Faculty of Veterinary Medicine, Universitas Airlangga (2.KE.049.04.2019). Fasting blood glucose levels were measured before and after lard and streptozotocin administration. All mice were in control of environmental conditions $\left(25 \pm 5^{\circ} \mathrm{C}\right.$, humidity of $50 \pm 10 \%$, and 12 light/dark cycles). Mice were provided with water and standard pellets 
ad libitum. Diabetes was induced in mice by multiple lowdose streptozotocin (STZ) with $30 \mathrm{mg} / \mathrm{kg}$ in citrate buffer $\mathrm{pH}$ 4 via injection intraperitoneally for five consecutive days following a high-fat diet for three weeks. Only mice with fasting blood glucose levels of more than $130 \mathrm{mg} / \mathrm{dL}$ were used as diabetic mice.

2.5. Animal Treatments. The research used a completely randomized design according to the previous studies $[12,14]$. Mice were divided into normal control $(\mathrm{KN})$ and diabetic groups. The diabetic group was further divided into three treatment groups: diabetic control (KD), S. duplicatum treatment group (PA), and a combination of $S$. duplicatum and G. mangostana treatment groups (PAM). Each group consisted of six mice. The treatment dose was $50 \mathrm{mg} / \mathrm{kg}$ of body weight. All mice were wounded by making a $1 \mathrm{~cm}$ section on the gluteal (under ethyl chloride local anesthesia), and this activity was considered day 0 . Each group has three treatment durations of 3 days, 7 days, and 14 days. At the end of the treatment, those mice were sacrificed, anesthetized by ketamine $(15 \mathrm{mg} / \mathrm{kg}$ body weight $)$ and xylazine $(2 \mathrm{mg} / \mathrm{kg}$ body weight) intramuscularly. Their skin was cut and proceeded for further histological observation.

2.6. Histological Observation. The histological observation was conducted on the cross section of wounded skin $(5 \mathrm{~mm}$ thickness, hematoxylin-eosin ( $\mathrm{H}$ and $\mathrm{E})$ staining). The wound healing process was determined by wound width, the number of neutrophils (polymorphonuclear cells, lobulated nucleus, and small granules), macrophages (large cell, round cell, round nucleus, or vacuolated cytoplasm to support phagocytic activity), fibroblasts (large flat, irregular shape cell, oval shape of nucleus, or basophilic cytoplasm), fibrocytes (elongated nuclei, spindle shape, or acidophilic cytoplasm), and collagen density at the wound-healing site. Wound width was measured under the light microscope at $40 \times$ magnification, while others at $400 \times$ magnification were observed at the connective tissue in the wound healing site. ImageJ software was used to measure wound width and collagen density.

2.7. Statistical Analysis. All data were presented as mean \pm SD and analyzed by one-way ANOVA followed by the Duncan test to evaluate significance between groups $(\alpha=0.05)$ with SPSS 21 software (SPSS Ltd., Surrey, UK). There was a significant difference while $p<0.05$.

\section{Results}

3.1. Effect of S. duplicatum and G. mangostana on Wound Healing Parameters. As shown in Table 1, on day 3 measurement, the diabetic control group (KD) showed a significant decrease $(p<0.05)$ in wound width, fibrocytes number, and collagen density in comparison with those of the normal control group (KN), but the group also showed a significant increase in neutrophils number and macrophages number. Inducing with $S$. duplicatum extract only (PA) exhibited significantly increased fibrocyte number and collagen density compared to those of the diabetic control group. Moreover, the $S$. duplicatum group also displayed a significant decrease in neutrophil numbers and macrophage numbers. In addition, when compared to the control group, the combination extracts group could significantly increase the fibrocytes number and collagen density in wound width while showing a significant decrease in neutrophils number and macrophages number. On day 7 , the diabetic control group significantly increased in the wound width's neutrophil number and macrophage number parameters and significantly decreased in fibroblast number, fibrocyte number, and collagen density compared to those of the normal control group. Otherwise, the $S$. duplicatum extract group showed a significant decrease in the neutrophils number and macrophages number in wound width compared to the diabetic control group. However, the fibrocytes numbers and collagen density parameters exhibited contrary results, which significantly increased the effect of the extract. On day 14, the diabetic control group displayed a significantly increased neutrophil number and a significantly decreased fibrocyte number only after being compared with the normal control group. Therefore, no significant report was present in between $S$. duplicatum group and the diabetic control group, whereas the combination extracts group (PAM) showed a significant increase $(p<0.05)$ in fibroblast number and fibrocyte number in comparison with those of the diabetic control group.

3.2. Effect of S. duplicatum and G. mangostana on Histology of the Wound Healing Site. As shown in Figure 1, this histological analysis displayed re-epithelization during the wound healing process. On day 3 , the $\mathrm{KN}$ group with no diabetic wound showed a fairly wide wound within the epidermis. Meanwhile, in the KD group has a wound diameter slightly wider than the $\mathrm{KN}$ group. The PA group displayed a tighter size diameter than the KD group, which is almost similar to the KN group. After that, the PAM group presented better treatment in reducing the diameter of the wound compared to other groups. On day 7, the wound diameter in the KD group was still large, whereas in another group showed improvement with a tighter size diameter. However, all groups showed equally well-wound closure on day 14 .

3.3. Effect of S. duplicatum and G. mangostana on Histology of Connective Tissue. These results were related to connective tissue attribute numbers such as neutrophil, macrophage, fibroblast, fibrocyte, and collagen density, as shown in Figure 2. On day 3, the KD group presented the most increasing neutrophil and macrophage cell numbers when the healing process was still at the first stage, but fibroblast, fibrocyte, and collagen density were still less than other groups. On day 7, all groups had decreasing neutrophil and macrophage cells, with a still high number in the KD group. The fibroblasts, fibrocytes, and density collagen displayed an increase in numbers than before, whereas the PAM group gave the best growth. On day 14, all groups already produced collagen in the almost totally wounded area and massively 
TABLE 1: Wound healing parameters on day 3, 7, and 14 of treatment.

\begin{tabular}{|c|c|c|c|c|c|c|c|c|}
\hline & Groups & & $\begin{array}{l}\text { Wound width } \\
\qquad(\mu \mathrm{m})\end{array}$ & $\begin{array}{l}\text { Neutrophils } \\
(\text { cells/nm²) }\end{array}$ & $\begin{array}{l}\text { Macrophage } \\
\left(\text { cells } / \mathrm{nm}^{2}\right)\end{array}$ & $\begin{array}{l}\text { Fibroblast } \\
\left(\text { cells } / \mathrm{nm}^{2}\right)\end{array}$ & $\begin{array}{l}\text { Fibrocyte } \\
\left(\text { cells/nm }{ }^{2}\right)\end{array}$ & $\begin{array}{c}\text { Collagen density } \\
(\%)\end{array}$ \\
\hline \multirow{3}{*}{$\mathrm{KN}$} & Day & 3 & $4849 \pm 106.07$ & $117.5 \pm 5.42$ & $12.5 \pm 1.18$ & $46.17 \pm 7.78$ & $21.33 \pm 1.41$ & $63.5 \pm 4.01$ \\
\hline & Day & 7 & $1166.17 \pm 20.03$ & $88.17 \pm 1.18$ & $8.83 \pm 0.24$ & $73.67 \pm 3.3$ & $28.5 \pm 1.65$ & $80.83 \pm 1.18$ \\
\hline & Day & 14 & $206.83 \pm 7.78$ & $31.5 \pm 0.71$ & $4.67 \pm 0.47$ & $45.83 \pm 0.24$ & $16.83 \pm 1.18$ & $93 \pm 1.41$ \\
\hline \multirow{3}{*}{$\mathrm{KD}$} & Day & 3 & $2642.17 \pm 33.71^{*}$ & $159.83 \pm 6.36^{*}$ & $17 \pm 0.94^{*}$ & $41.67 \pm 10.84$ & $13.17 \pm 0.71^{*}$ & $56.67 \pm 1.89^{*}$ \\
\hline & Day & 7 & $6478.5 \pm 320.32^{*}$ & $132.67 \pm 7.07^{*}$ & $15.83 \pm 4.01^{*}$ & $51.83 \pm 9.19^{*}$ & $19.5 \pm 0.71^{*}$ & $60.83 \pm 4.01^{*}$ \\
\hline & Day & 14 & $243.17 \pm 10.61$ & $57.83 \pm 6.84^{*}$ & $2.67 \pm 0.47$ & $38.83 \pm 4.95$ & $14 \pm 0.94^{*}$ & $89.67 \pm 1.41$ \\
\hline \multirow{3}{*}{$\mathrm{PA}$} & Day & 3 & $3374.5 \pm 381.6^{*}$ & $115.5 \pm 7.31^{+}$ & $11 \pm 0.94^{+}$ & $35.33 \pm 1.89$ & $17 \pm 0.94^{*+}$ & $66.67 \pm 0.94^{+}$ \\
\hline & Day & 7 & $1565 \pm 447.83^{+}$ & $98.5 \pm 8.72^{+}$ & $9.17 \pm 0.71^{+}$ & $60.17 \pm 3.54^{*}$ & $26.67 \pm 0.47^{+}$ & $87.5 \pm 1.18^{*+}$ \\
\hline & Day & 14 & $162.33 \pm 17.91$ & $61.17 \pm 24.75^{*}$ & $2.17 \pm 0.24$ & $41.83 \pm 1.18$ & $14.5 \pm 0.24^{*}$ & $90.5 \pm 0.71$ \\
\hline \multirow{3}{*}{ PAM } & Day & 3 & $4701.67 \pm 68.35^{+}$ & $131.67 \pm 9.43^{+}$ & $11.83 \pm 0.71^{+}$ & $35.17 \pm 4.01$ & $21.83 \pm 0.71^{+}$ & $68.83 \pm 0,71^{*+}$ \\
\hline & Day & 7 & $1512.83 \pm 27.11^{+}$ & $114.83 \pm 9.66^{*}$ & $8.67 \pm 0.47^{+}$ & $67.83 \pm 0.71^{*+}$ & $30.5 \pm 1.18^{+}$ & $82.17 \pm 2.59^{*+}$ \\
\hline & Day & 14 & $136.5 \pm 4.01$ & $48.17 \pm 0.24$ & $1.83 \pm 0.24$ & $56 \pm 7.07^{+}$ & $17.33 \pm 1.41^{+}$ & $93.83 \pm 2.12$ \\
\hline
\end{tabular}

Note: The data are expressed as the mean $\pm \mathrm{SD}(n=6) .{ }^{*} p<0.05$ compared to the normal control group. ${ }^{+} p<0.05$ compared to the diabetic control group. KN: normal control, KD: diabetic control, PA: S. duplicatum extract treatment, and PAM: combination of S. duplicatum and G. mangostana extracts treatments.
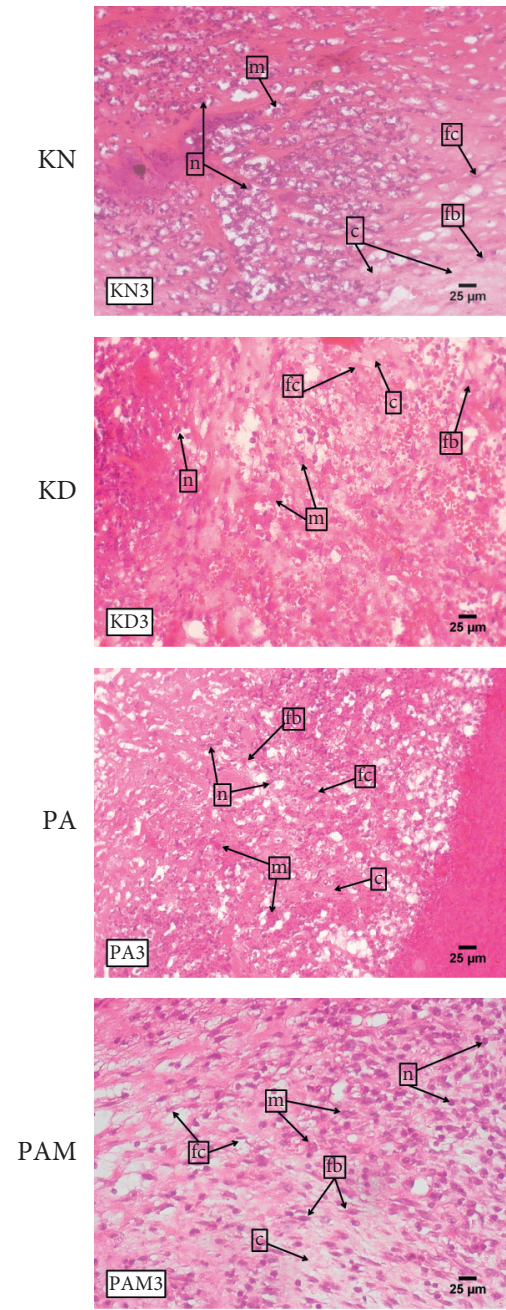
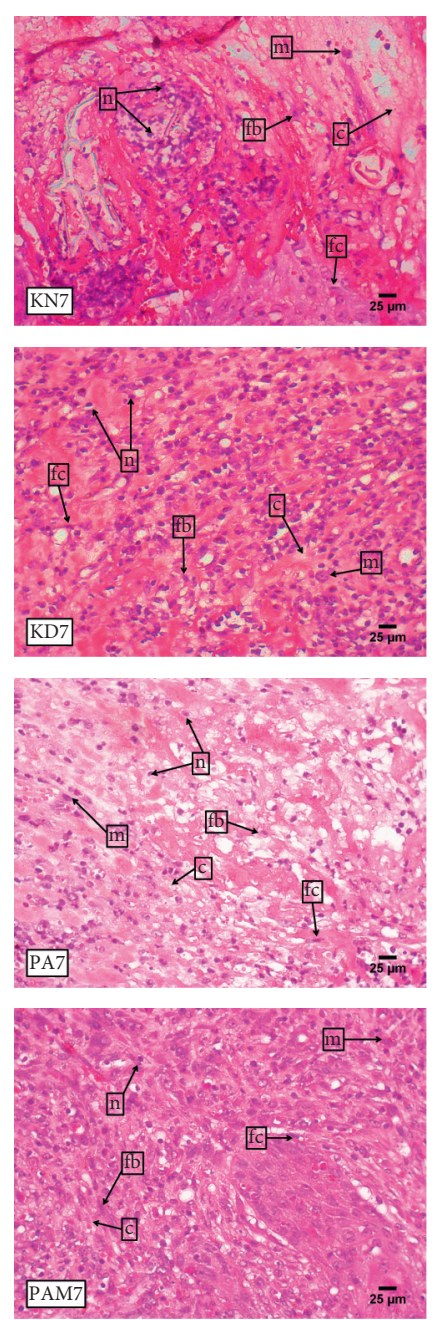
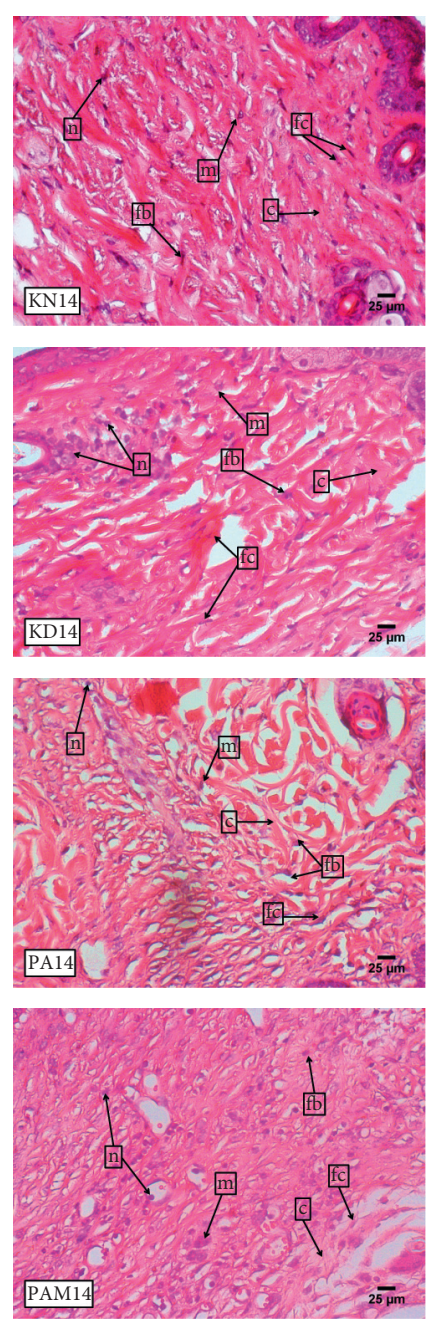

Figure 1: Wound healing parameters on day 3, 7, and 14 of treatment. Wound width (a), the number of neutrophils (b), the number of macrophages (c), the number of fibroblasts (d), the number of fibrocytes (e), and collagen density (f) in the open wound healing process in diabetic mice. Different letters show a significant difference based on the Duncan test $(\alpha<0.05)$. KN: normal control, KD: diabetic control, PA: S. duplicatum extract treatment, and PAM: combination of S. duplicatum and G. mangostana extract treatment. 

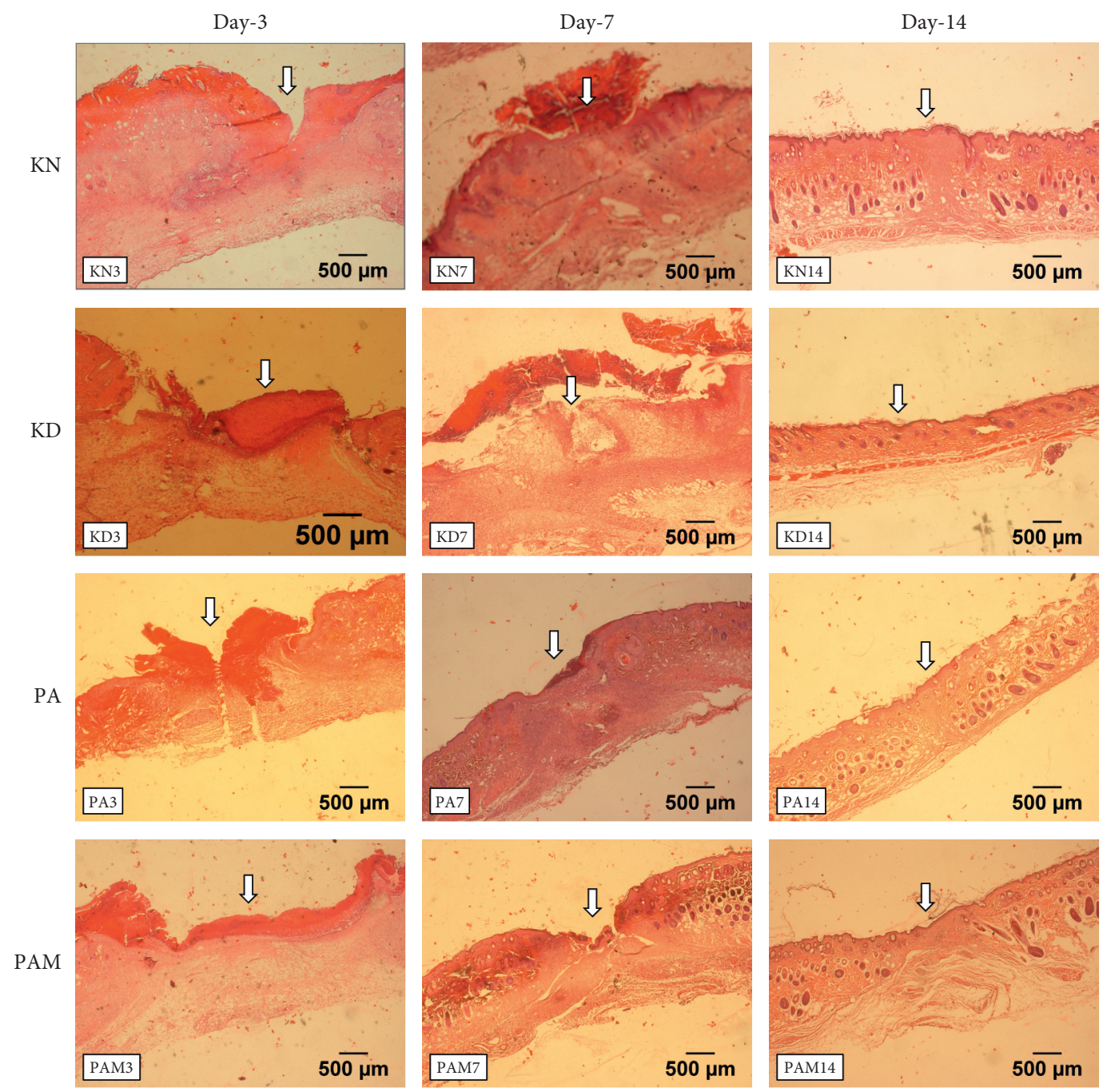

FIGURE 2: Histology of the wound healing site (pointed by white arrows). KN: normal control, KD: diabetic control, PA: S. duplicatum extract treatment, and PAM: the combination of S. duplicatum and G. mangostana extract treatment.

decreased neutrophil, macrophage, fibroblast, and fibrocyte numbers in that wound area.

\section{Discussion}

Diabetic wounds are often experienced by many diabetic patients and are called the silent killers due to the difficulty of handling these wounds. Inflammatory cells are linked with wound healing mechanisms in the skin, such as neutrophils and macrophages. Both are associated with phagocytosis. After neutrophils have reached the end of their lifetime in tissue, they would be cleansed mostly by macrophages through the phagocytosis process [20]. In this study, neutrophils play an essential role in wound healing, producing lysozyme enzymes, and cleaning bacteria and various cell debris [5]. Neutrophils also play an essential role in producing cytokines such as TNF- $\alpha$ and IL-10 and growth factors such as VEGF, IGF-1, and TGF- $\beta$ [6]. At 48 hours after the injury, the monocytes from the blood vessels around the wound move to the wound area and differentiate into macrophages activated through chemokine signaling.
Monocytes are differentiating into macrophages and can help neutrophils in phagocytosis [21]. The monocyte, which turns into macrophages, is a significant producer of proinflammatory cytokines that stimulate the migration and proliferation of fibroblasts, endothelial cells, and keratinocytes to repair skin tissue damage $[6,13]$. In the proliferation phase, fibroblasts are the primary cells in the tissue reconstruction process due to their ability to produce a certain amount of collagen needed in wound healing. Usually, fibroblasts are not active cells with metabolic activity and a proliferation rate, which tends to be slow. After the injury process, fibroblasts will become active cells proliferating rapidly and migrating to enter the injury area.

This result exhibited a proliferation process that becomes fibroplasia and produces collagen for reconstruction tissue [5]. Moreover, collagen synthesis is effective starting from day 7 after the injury. After that, the re-epithelialization process, which covers most of the keratinocytes, begins to migrate and experience stratification and differentiation to restructure the epidermal barrier function. The re-epithelialization process also increases extracellular matrix 
production, growth factors, cytokines, and angiogenesis through the release of growth factors such as keratinocyte growth factor [22]. The wound is healed after being supported by re-epithelialization. Diabetic conditions produce a severe infection, attracting more neutrophils and macrophages to clean debris cells and fight bacteria in the wound area. This mechanism is strengthened by infection due to high levels of glucose. In addition, neutrophils and macrophages will phagocyte bacteria, pathogens, and debris on the wound area, raising the number of both cells relatively higher than normal. The presence of high ROS levels caused a permanent hypoxic condition in the wound area. In addition, hyperglycemia and oxidative stress due to diabetes cause epigenic code mutations, resulting in polarization and macrophage modulation. The inability of macrophage polarization is one of the main reasons the wound healing process becomes postponed [8]. Diabetic conditions also inhibit the proliferation phase of the open wound healing process.

Excessive production of proinflammatory cytokines due to increasing ROS can inhibit the migration and proliferation of keratinocytes and fibroblasts and inhibit the synthesis of growth factors so that wound healing will be disrupted. Proinflammatory cytokine production due to increasing ROS causes angiogenic response disorders, microvascular complications, and disruption of macrophage and neutrophil functions $[9,10]$. Moreover, these cytokines are involved in the migration and proliferation of keratinocytes, fibroblasts, and growth factor production. High levels of proinflammatory cytokines such as IL- 6 and TNF- $\alpha$ in diabetes cause disruption of the inflammatory cascade, hyper inflammation, and insulin resistance [7]. Decreasing growth factors will decrease the proliferation and migration of fibrocytes and fibroblast cells. Hyperglycemia in people with diabetes also triggers active PKC pathways, which can inhibit the expression of eNOS and increase the expression of NF- $\kappa$ B [4].

The decreased expression of eNOS inhibits the migration of keratinocytes, thereby inhibiting the re-epithelization process [11]. It suggests that the re-epithelization in people with diabetes was impaired due to increased ROS caused by hyperglycemia. Besides the increased ROS, the disruption of the re-epithelization process is also caused by infection from bacteria. The process indicates that in diabetics, ROS will cause the growth factor-producing cells to malfunction. S. duplicatum can absorb excess wound exudate, maintain a physiologically humid environment, and minimize bacterial infections in the wound area. However, it does not have a maximal effect because the number of neutrophils is higher than the average at day-14, indicating there is still higher activity to bacteria phagocytosis. The antimicrobial capacity of neutrophils is higher than that of macrophages $[12,23]$. Therefore, these conditions give more effect to collagen synthesis only on the last healing process. The results showed that the infection is reduced in the PA group and does not disturb the proliferation phase significantly. Thus, the wound closure process using $S$. duplicatum improves the mechanism of angiogenesis and re-epithelization [24]. In addition, S. duplicatum has strong absorption and absorbs a lot of rapid exudates in a form of a gel that creates a moist environment and maintains the homeostatic condition of a wound to stimulate wound healing faster $[12,25]$. The numbers of neutrophils and macrophages are not significantly different from normal control. It showed that the combination of S. duplicatum and G. mangostana has potential antimicrobial, antioxidant, and anti-inflammatory properties [26]. The antioxidants in G. mangostana reduce ROS and decrease the inhibition for migration and proliferation of fibroblasts, endothelial cells, and keratinocytes, and repair skin tissue better than diabetic conditions [12]. The results displayed that xanthone compounds can reduce cell toxicity and damage by preventing NF- $\kappa$ B pathways and MAPK activation so that the re-epithelization proceeds smoothly [27]. Fibroblasts are the primary cells in the tissue reconstruction process in the proliferation phase due to collagen production needed for wound healing. There is a high possibility that the diabetic open wounds treated in combination with S. duplicatum and G. mangostana will have entirely healed before day 14 after the injury. Therefore, it is recommended to observe in 10-12 days after the injury for further research.

\section{Conclusions}

The topical administration of alginate extracted from Sargassum duplicatum and Garcinia mangostana combination can lead to re-epithelialization in the open wound of diabetic mice. Therefore, it can be concluded that the topical administration of alginate extracted from Sargassum duplicatum and Garcinia mangostana combination improved open wound healing in diabetic mice due to the antioxidants from xanthone and the polysaccharide polymer in alginate, which plays the role as an absorbent. Although the combination showed an improvement in open wound therapy, further study needs advanced methods, comparison with commercial drugs, and advanced variables such as NF- $\kappa \mathrm{B}$ and MAPK pathways to confirm the effectiveness of the Sargassum duplicatum and Garcinia mangostana combination.

\section{Data Availability}

The data used to support this study are available from the corresponding author on reasonable request.

\section{Conflicts of Interest}

The authors declare that there are no conflicts of interest.

\section{Acknowledgments}

The authors would like to thank the Directorate of Research and Community Service, Deputy of Strengthening Research and Development, Ministry of Research \& Technology for Research funding (483/UN3.15/PT/2021). We also appreciate Dean of Faculty of Science and Technology and Head of Research and Community Services of Universitas Airlangga for the opportunity to conduct this study. This manuscript is dedicated to the memory of Dr. Saikhu Akhmad Husen who sadly passed away on Saturday 17th July, 2021. 


\section{References}

[1] American Diabetes Association, "Diagnosis and classification of diabetes mellitus," Diabetes Care, vol. 36, no. Suppl 1, pp. S13-S27, 2013.

[2] S. Song, B. Wang, X. Zhang et al., "Long-term diabetes mellitus is associated with an increased risk of pancreatic cancer: a meta-analysis," PLoS One, vol. 10, no. 7, p. e0134321, 2015.

[3] C. M. O. Volpe, P. H. Villar-Delfino, P. M. F. Dos Anjos, and J. A. Nogueira-Machado, "Cellular death, reactive oxygen species (ROS) and diabetic complications," Cell Death \& Disease, vol. 9, no. 2, p. 119, 2018.

[4] O. M. Ighodaro, "Molecular pathways associated with oxidative stress in diabetes mellitus," Biomedicine \& Pharmacotherapy, vol. 108, pp. 656-662, 2018.

[5] A. C. d. O. Gonzalez, T. F. Costa, Z. d. A. Andrade, and A. R. A. P. Medrado, "Wound healing-a literature review," Anais Brasileiros De Dermatologia, vol. 91, no. 5, pp. 614-620, 2016.

[6] S. Babaei, M. Bayat, M. Nouruzian, and M. Bayat, "Pentoxifylline improves cutaneous wound healing in streptozotocininduced diabetic rats," European Journal of Pharmacology, vol. 700, no. 1-3, pp. 165-172, 2013.

[7] S. Patel, S. Srivastava, M. R. Singh, and D. Singh, "Mechanistic insight into diabetic wounds: pathogenesis, molecular targets and treatment strategies to pace wound healing," Biomedicine \& Pharmacotherapy, vol. 112, p. 108615, 2019.

[8] S. B. Mallik, B. S. Jayashree, and R. R. Shenoy, "Epigenetic modulation of macrophage polarization-perspectives in diabetic wounds," Journal of Diabetes and its Complications, vol. 32, pp. 524-530, 2019.

[9] A. Alavi, R. G. Sibbald, D. Mayer et al., "Diabetic foot ulcers: part I. pathophysiology and prevention," Journal of the American Academy of Dermatology, vol. 70, no. 1, pp. 1-20, 2014.

[10] S.-i. Okizaki, Y. Ito, K. Hosono et al., "Suppressed recruitment of alternatively activated macrophages reduces TGF- $\beta 1$ and impairs wound healing in streptozotocin-induced diabetic mice," Biomedicine \& Pharmacotherapy, vol. 70, pp. 317-325, 2015.

[11] H. Brem and M. Tomic-Canic, "Cellular and molecular basis of wound healing in diabetes," Journal of Clinical Investigation, vol. 117, no. 5, pp. 1219-1222, 2007.

[12] Z. N. Ilmi, P. A. C. Wulandari, S. A. Husen et al., "Characterization of alginate from Sargassum duplicatum and the antioxidant effect of alginate-okra fruit extracts combination for wound healing on diabetic mice," Applied Sciences, vol. 10, no. 17, p. $6082,2020$.

[13] B. Aderibigbe and B. Buyana, "Alginate in wound dressings," Pharmaceutics, vol. 10, no. 2, p. 42, 2018.

[14] P. A. C. Wulandari, Z. N. Ilmi, S. A. Husen et al., "Wound healing and antioxidant evaluations of alginate from Sargassum ilicifolium and mangosteen rind combination extracts on diabetic mice model," Applied Sciences, vol. 11, no. 10, p. $4651,2021$.

[15] W. Widowati, D. R. Laksmitawati, T. L. Wargasetia et al., "Mangosteen peel extract (Garcinia mangostana L.) as protective agent in glucose-induced mesangial cell as in vitro model of diabetic glomerulosclerosis," Iranian Journal of Basic Medical Sciences, vol. 21, no. 9, pp. 972-977, 2018.

[16] W. Suttirak and S. Manurakchinakorn, "In vitro antioxidant properties of mangosteen peel extract," Journal of Food Science and Technology, vol. 51, no. 12, pp. 3546-3558, 2014.
[17] T. Fu, S. Wang, J. Liu et al., "Protective effects of $\alpha$-mangostin against acetaminophen-induced acute liver injury in mice," European Journal of Pharmacology, vol. 827, pp. 173-180, 2018.

[18] A. Mazumder, S. L. Holdt, D. De Francisci, M. AlvaradoMorales, H. N. Mishra, and I. Angelidaki, "Extraction of alginate from Sargassum muticum: process optimization and study of its functional activities," Journal of Applied Phycology, vol. 28, no. 6, pp. 3625-3634, 2016.

[19] A. Ghasemzadeh, H. Jaafar, A. Baghdadi, and A. TayebiMeigooni, "Alpha-mangostin-rich extracts from mangosteen pericarp: optimization of green extraction protocol and evaluation of biological activity," Molecules, vol. 23, no. 8, p. 1852, 2018.

[20] D. L. Bratton and P. M. Henson, "Neutrophil clearance: when the party is over, clean-up begins," Trends in Immunology, vol. 32, no. 8, pp. 350-357, 2011.

[21] S. A. Eming, T. Krieg, and J. M. Davidson, "Inflammation in wound repair: molecular and cellular mechanisms," Journal of Investigative Dermatology, vol. 127, no. 3, pp. 514-525, 2007.

[22] I. Pastar, O. Stojadinovic, N. C. Yin et al., "Epithelialization in wound healing: a comprehensive review," Advances in Wound Care, vol. 3, no. 7, pp. 445-464, 2014.

[23] M. T. Silva and M. Correia-Neves, "Neutrophils and macrophages: the main partners of phagocyte cell systems," Frontiers in Immunology, vol. 3, p. 174, 2012.

[24] K. E. Johnson and T. A. Wilgus, "Vascular endothelial growth factor and angiogenesis in the regulation of cutaneous wound repair," Advances in Wound Care, vol. 3, no. 10, pp. 647-661, 2014.

[25] W. Yu, Y.-Y. Jiang, T.-W. Sun et al., "Design of a novel wound dressing consisting of alginate hydrogel and simvastatin-incorporated mesoporous hydroxyapatite microspheres for cutaneous wound healing," RSC Advances, vol. 6, pp. 104375-104387, 2016.

[26] S. R. Yende, U. N. Harle, and B. B. Chaugule, “Therapeutic potential and health benefits of Sargassum species," Pharmacognosy Reviews, vol. 8, no. 15, pp. 1-7, 2014.

[27] N. Tatiya-Aphiradee, W. Chatuphonprasert, and K. Jarukamjorn, "In vivo antibacterial activity of Garcinia mangostana pericarp extract against methicillin-resistant Staphylococcus aureus in a mouse superficial skin infection model," Pharmaceutical Biology, vol. 54, no. 11, pp. 2606-2615, 2016. 